\title{
Principles Developed for the Construction of the High Performance, Low-Cost Superconducting LHC Corrector Magnets
}

\author{
A. Ijspeert, M. Allitt, A. Hilaire, M. Karppinen, J. Mazet, J. Perez, J. Salminen, M. Karmarkar, and A. Puntambekar
}

\begin{abstract}
The Large Hadron Collider (LHC) needs more than 6000 superconducting corrector magnets. These must be sufficiently powerful, have enough margin, be compact and of low cost. The development of the 11 types of magnets was spread over several years and included the magnetic and mechanical design as well as prototype building and testing. It gradually led to the systematic application of a number of interesting construction principles that allow to realize the above mentioned goals. The paper describes the techniques developed and presently used in practically all the $\mathrm{LHC}$ corrector magnets ranging from dipoles to dodecapoles.
\end{abstract}

Index Terms-Accelerator magnet construction, Large Hadron Collider, superconducting corrector magnets.

\section{INTRODUCTION}

$\mathbf{T}$ HE LARGE Hadron Collider (LHC) will be equipped with corrector magnets ranging from dipoles, quadrupoles and sextupoles to octupoles, decapoles and dodecapoles. These magnets (Table I) are necessary for the correction of field errors and for the control and steering of the beams. The development work concentrated on combining high field strength and good field quality with a compact design, featuring low nominal currents and economic construction methods. Typically the peak fields are of the order of $3 \mathrm{~T}$ in a bore of $56 \mathrm{~mm}$ diameter. The outer diameters, including the iron yokes, are less than 194 $\mathrm{mm}$ (the distance of separation between the two beams) so just over three times the bore diameter. The nominal currents range from $55 \mathrm{~A}$ to $550 \mathrm{~A}$. The paper explains the principles that were developed, tested on prototypes, and applied on practically all the correctors to achieve these objectives.

\section{The MAgnet CONSTRUCTION}

\section{A. Construction Principles and Number of Coils}

The magnets are built in the form of modules each of which is a complete magnet. These modules are then centered with keys in a support structure to ensure alignment with the two parallel beams. The typical magnet is composed of a number of coils wound from enameled monolithic conductors and resin

Manuscript received September 24, 2001.

A. Ijspeert, M. Allitt, A. Hilaire, M. Karppinen, J. Mazet, and J. Perez are with CERN, European Organization for Nuclear Research, 1211 Geneva 23, Switzerland.

J. Salminen was with CERN. He is now with Nokia, Helsinki, Finland.

M. Karmarkar and A. Puntambekar are with CAT, Centre for Advanced Technology, Indore, India.

Publisher Item Identifier S 1051-8223(02)03436-X.
TABLE I

LiST OF CORRECTOR MAGNET MODULES (Not INCLUding EXTRA COIL INSERTS)

\begin{tabular}{|c|c|c|c|c|c|c|c|c|c|c|c|}
\hline \multirow[t]{2}{*}{ NQWE } & \multirow{2}{*}{$\begin{array}{l}\text { Prie } \\
\text { Ham } \\
\text { n }\end{array}$} & \multirow{2}{*}{$\begin{array}{r}\text { RONBER } \\
\text { baty }\end{array}$} & \multicolumn{3}{|c|}{ GMERSICN8 } & \multicolumn{2}{|c|}{ MESETE } & \multicolumn{2}{|c|}{ ELECIFOC } & \multicolumn{2}{|l|}{ Whe } \\
\hline & & & 10. & $\begin{array}{l}0.0 \\
\text { inm }\end{array}$ & 1 & Bued & $n p$ & Corest & moat & Secten & Case \\
\hline MCD & 5 & 752 & 94 & 192 & 73 & 20 & si & s5 & 7000 & $0=0.375$ & \& \\
\hline MCnC & 1 & 158 & $9 s$ & 997 & 1450 & 3.1 & 57 & 100 & 2000 & 0.30 .0057 & \\
\hline $\mathrm{mct}$ & 1 & as & $\pi$ & 197 & 1900 & 25 & $\omega$ & $n 2$ & $\sin$ & 0.20 .095 & 4 \\
\hline Mctix & 1 & 27 & $\mathbf{n}$ & 350 & $\pi 00$ & 1.4 & so & 550 & 200 & $0.86 \times 1.53$ & 16 \\
\hline & 2 & & s. & 135 & 380 & 4 & $\$ 2$ & 950 & an & 0.6 & 10 \\
\hline Mor & 2 & 112 & st & 135 & 1486 & 3.5 & 57 & 450 & 1200 & $0.61 \times 1.13$ & 1.6 \\
\hline MOSx & 2 & ક & $n$ & $t a 0$ & 596 & 2.2 & 52 & 590 & 14 & $0.61 \times 1.13$ & 1.8 \\
\hline & 3 & 264 & 59 & 96 & 160 & 14 & 30 & 59 & 0 a & 1.13 & 16 \\
\hline WE & 3 & 752 & 34 & เ92 & 465 & 35 & 54 & 390 & 39 & $061 \times 1.13$ & 16 \\
\hline$M O$ & 4 & $\mathbf{x t}$ & 9 & 104 & 390 & 13 & 49 & 560 & 1.5 & $0.61 \times 1.13$ & 16 \\
\hline MCD & 3 & 1232 & 64 & 101 & 192 & 13 & 30 & 250 & D.4 & $0.61 \times 1.13$ & 1.6 \\
\hline
\end{tabular}

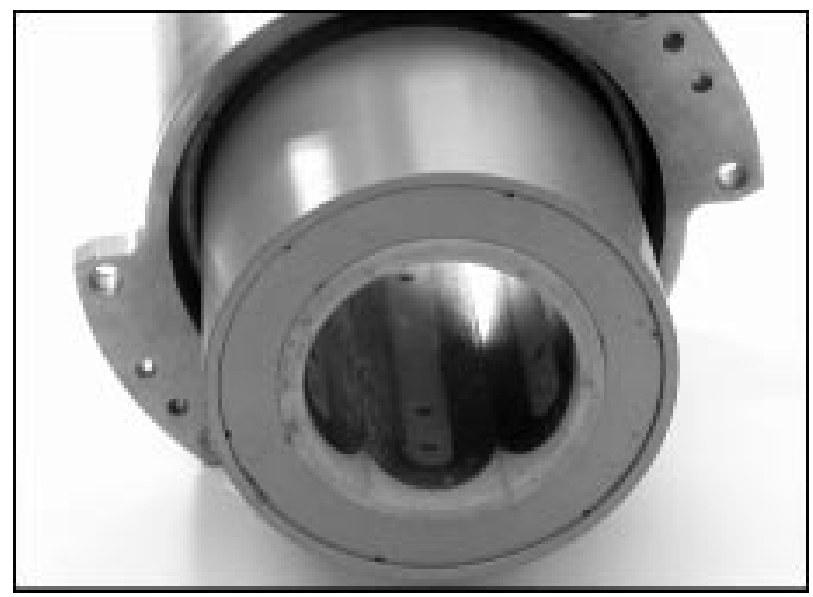

Fig. 1. Typical cross section of a corrector magnet (MCS sextupole).

impregnated. These coils are assembled on a cylindrical surface (Fig. 1), packed in a layer of pre-preg insulation, slipped into iron yoke laminations and pre-stressed by means of aluminum shrink-rings. The usual number of coils per magnet is " $2 n$ " but for the magnets of an order higher than sextupole the magnets have been constructed with only " $n$ " coils [1], [2].

\section{B. Choice of Current Level}

The design current should be as low as possible to minimize the cost of the power-supplies, the "warm" cables and the current leads. This is in particular the case for individually powered magnets where the cost of these items can easily exceed the cost of the superconducting magnets they feed. Another reason to go for low current is to reduce the heat leak into the cryostat through the current leads. However, small currents make it necessary to design coils with many turns resulting in high inductances. A limit is reached when the magnet inductance causes 


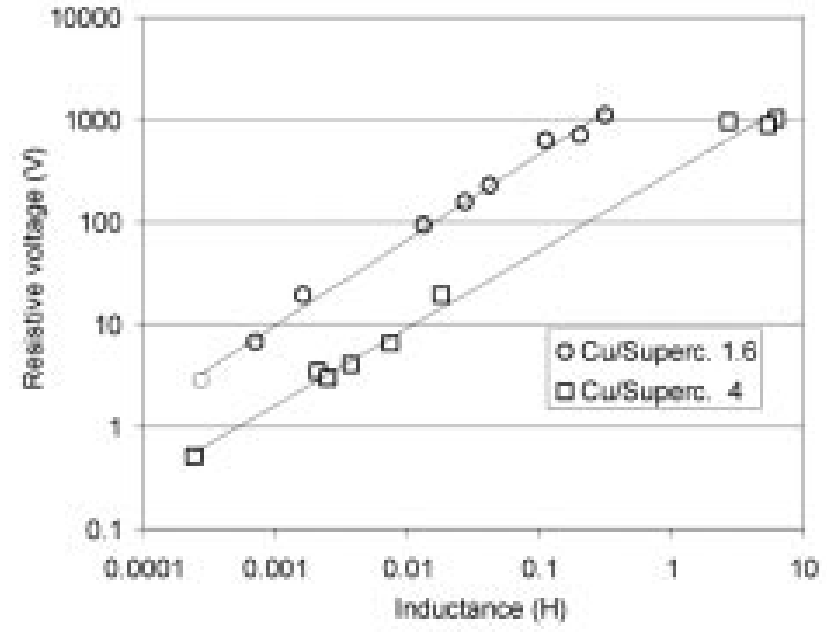

Fig. 2. Calculated resistive peak voltages for the 18 magnets (modules and inserts) as a function of their inductance. Grouped by the copper-to-superconductor ratios of their wires. Quench at nominal currents.

too high quench voltages and creates a risk for the electrical safety of the magnet. Fig. 2 shows the resistive quench voltages calculated for all the 18 types of coils we use in the 11 different corrector types (quench at nominal currents). Four types of wire are being used and the data have been grouped by their copper-to-superconductor ratios, 1.6 and 4. It can be seen that the voltages grow quasi linearly with the inductance. The wires with 2.5 times more copper show voltages reduced by practically a factor of 10 . It should be noted that the voltages as measured on the coils are lower because the inductive voltage of the quenching coil compensates part of the resistive coil voltage. Prototype tests showed that the individually powered orbit correctors in the arc of $1.5 \mathrm{Tm}$ integrated strength could be run at 55 A. Going to more turns and lower current appeared too risky; on a first prototype designed for 30 A nominal current we measured coil voltages as high as $800 \mathrm{~V}$. The stronger individually powered orbit correctors in the insertion regions $(2.5 \mathrm{Tm})$ could not be made for 55 A but 110 A was safe. For the magnets connected in series the cost of the "warm" powering counts less and therefore a less critical current level of 550 A was chosen for practically all those magnets. The peak temperature reached during a quench is a function of many magnet parameters. However for a given magnet it is independent of the choice of wire size as long as the current density and the composition of the wire are kept the same. Each individual magnet has been designed to be able to absorb its own energy without heating by more than $200 \mathrm{~K}$ whereas the magnets connected in families are protected by means of a parallel resistor over each magnet in addition to energy extraction.

\section{CoIl CONSTRUCTION}

\section{A. Counter Winding}

The "counter-winding" method was developed to minimize the amount of coil winding labor and time [1]. The first feature was to limit the number of layers to only two and wind these not up and down but spiraling outwards from the central post. The absolute minimum of just one layer jump is then needed to

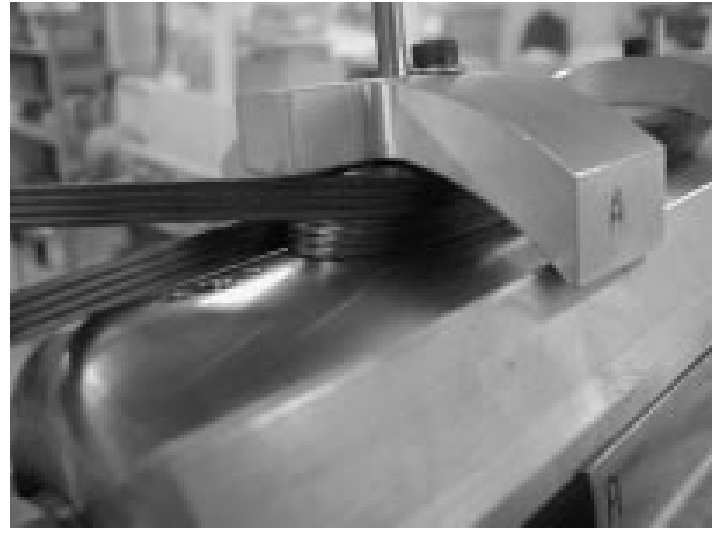

Fig. 3. Counter-winding a coil with a flat cable (MS sextupole model).

radially go from the inner to the outer layer. This jump can be made at the start of the winding or be prepared before bringing the wire to the winding mandrel.

The second feature was to wind these two layers simultaneously starting from the layer jump placed at the end of the central post. The lower layer is wound spiraling the wire outwards by turning in one direction around the central post and the upper layer spiraling the wire simultaneously outwards but turning in the opposite direction [3]. The cylindrical surfaces of the winding mandrel on the inner radius and of a screen fixed for this purpose on the outer radius force the two layers to take their position side by side. This makes it superfluous to use tooling to hold every winding in place and clears the way to fast and even automatic winding. The coil ends come straight out of the coil and need no extra bends.

In most of the cases the coils are "wet wound," using epoxy glue, and then molded and cured. The precise coil dimensions are obtained thanks to molding using precise tooling.

\section{B. Flat Cable}

For the stronger corrector magnets like the Tuning Quadrupoles (MQT and MQTL) and Chromaticity Sextupoles (MS) two layers of wire are not sufficient and typically 6 and 8 layers are necessary. Conventionally such coils are wound in layers winding upward or downward along the edge of the central post, every new layer being wound around the earlier one. However this is time consuming due to the many layer jumps and also because every turn needs to be kept in position. In addition, expensive end spacers are needed between each layer to house the outward layer jumps. To avoid this the counter-winding principle was applied. Two solutions have been tried. The first one is to superpose a number of counter-wound double layer coils of the type described before and connect them in series. The second is to counter-wind just one double layer coil but now using instead of the single wire a flat cable composed of several wires glued in parallel, like already experienced in [4], again making serial connections at the end. Such cables appeared to be very tough and to accept all kinds of bends and torsions.

Both solutions worked well [5]. The second solution, using the flat cable, has been adopted for the fabrication of the final magnets (Fig. 3). It has the advantage that there are less coils to 


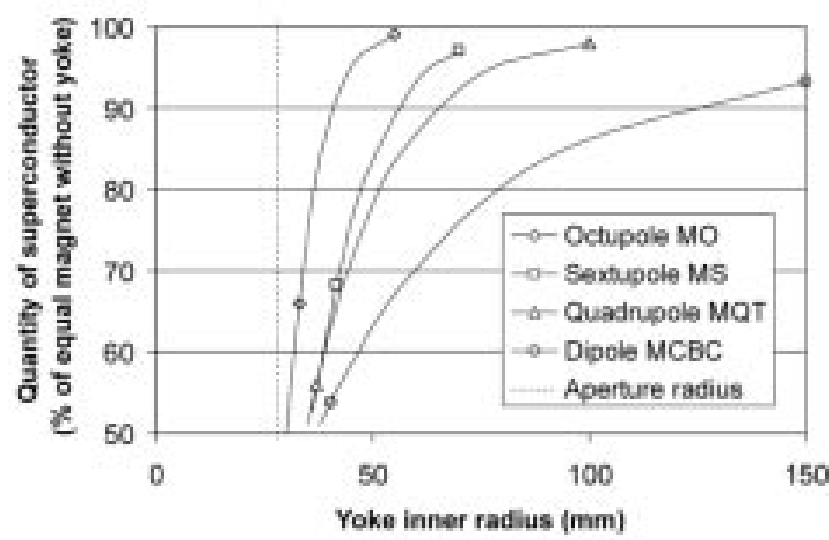

Fig. 4. Necessary amount of superconductor as a function of the inner radius of the iron yoke (100\% when iron yoke at infinity). Magnet current and working point maintained.

be wound, and less parts to be made (central posts) whereas the number of interconnections is the same as for the other solution. The connections are made outside the coils on a compact endflange using ultrasonic welding, a method that allows to obtain a low contact resistance, 5 times lower than a soldered connection of the same length, while being fast and reliable.

\section{Suppression of End-Spacers}

The "counter-wound" coils have no end-spacers, each coil is wound as a single coil block. There are two reasons, one is the high cost of such spacers and the other the fact that spacers make the counter-winding more complicated. In a coil with internal spacers, the wire somewhere has to go from the last turn inside the spacers to the next turn outside the spacers. This is achieved cutting a path through the end-spacer. In the case of the counter-winding where we wind two layers at the same time the two wires will cut along different paths through the end spacers. This means that one needs different endspacers for each of the two layers. We did not wish to go to such complications and designed all the multipole coils as a single block. As a consequence, optimizing the angles allowed suppression of the first higher order "allowed" multipole but not of the second higher order "allowed" multipole, but we could accept this drawback.

Note that if one only introduces spacers in the straights and not in the ends or inversely only in the ends but not in the straights this problem disappears and this may be a way for correction of the second higher order multi-pole in counter-wound coils.

End-spacers, Besides correcting the field quality, also reduce the peak field in the coil ends and thus help lower the working point of the magnet, a feature our method does not profit from.

\section{Dipole Corrector Coils Made in a Single Layer}

The dipole correctors are individually powered and built with thin wire for $55 \mathrm{~A}$ and $110 \mathrm{~A}$. The coils consist typically of a thousand of turns with a 0.3 to $0.4 \mathrm{~mm}$ wire. Winding this in a regular pattern is nearly impossible and the flat cable principle is doubly advantageous. The wires are pre-assembled in the form of a ribbon of typically between 15 and 20 wires. It would have been attractive to wind these coils also using the

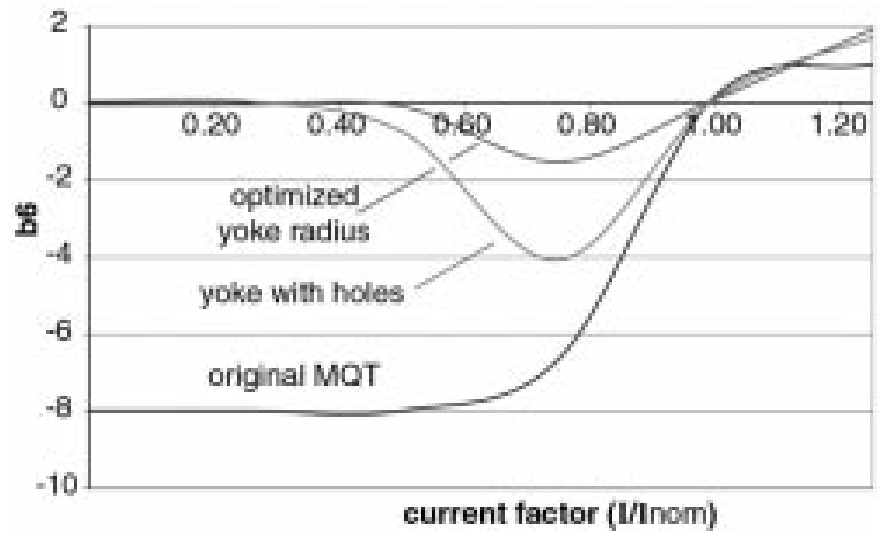

Fig. 5. Saturation effect on first harmonic (MQT quadrupole). Effect of holes and effect of optimized yoke outer diameter, respectively.

counter-winding technique. Since these dipoles need spacers in the straight and in the end for field quality the counter winding technique is not well applicable. The dipole coils are wound as a single layer of cable spiraling out from the central post, without layer jumps. They wind easily, incorporating the longitudinal and end spacers necessary for field quality control. There are two drawbacks. First, in order to start the first turn, the incoming cable must now pass over the coil end, enter into a space left in the central post where it must be twisted into an orientation necessary for the start of the turn. Second the number of serial connections is now double that in an identical counter-wound coil.

\section{YoKe ASSEMBLY}

\section{A. Iron Close to the Coil}

The iron yoke has been brought as close to the coil as possible, typically $2 \mathrm{~mm}$ from the outer radius of the coil, the space in between being taken by the ground insulation of the coil. One reason for this is to obtain very compact magnets and keep the outer diameter of the magnet modules within the $194 \mathrm{~mm}$ of beam separation. The second reason is to save on superconductor. Fig. 4 highlights the saving in superconductor as compared to equally strong magnets without iron yoke. It shows for the different multipole magnets the necessary amount of superconductor as a function of the inner radius of the iron yoke. A saving of about $50 \%$ is obtained for all types of magnet when the iron touches the coil. The figure also shows that for higher multi-pole magnets the iron effect diminishes quickly for larger iron radii. Our designs require only $70 \%$ of superconductor and some need as little as $55 \%$ as compared to equally strong magnets without iron yoke.

The ground insulation is put on in the form of pre-preg bandage or also by gluing a pre-machined split-tube of insulation material around the coil. The latter allows a faster and less complex assembly procedure.

\section{B. Reduction of Saturation Effects}

The iron close to the coil boosts the magnetic field but also introduces saturation effects when the fields rise above 2 Tesla. 


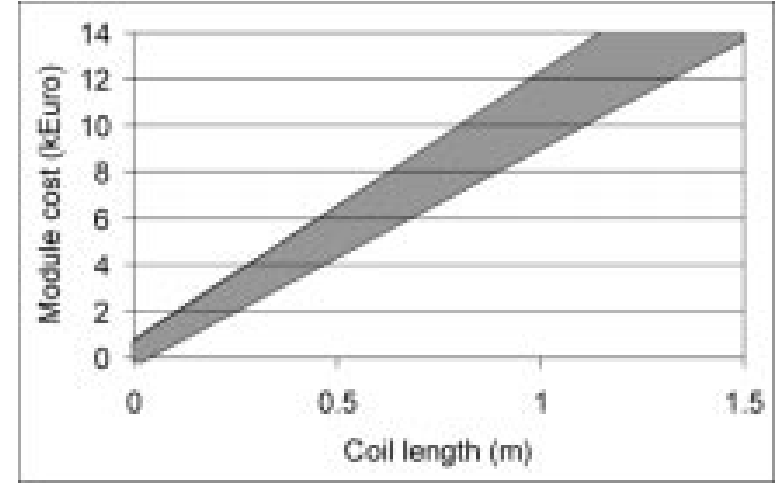

Fig. 6. Cost of the magnets (modules) as a function of their lengths. Cost includes materials, tooling, fabrication and cold testing $(4.2 \mathrm{~K})$.

This saturation causes the first "allowed" higher multipole to change with excitation deteriorating the optimized field quality. However there are ways to reduce this effect. One is to introduce in the laminations holes that redistribute the flux in the iron [6]-[7]. Done in a sophisticated way this can reduce the saturation effects for several harmonics. Another method is to optimize the width of the iron yoke that allows to reduce the effect for the first harmonic. The second method has been applied for the series quadrupoles and sextupoles. Fig. 5 shows calculation results for both methods applied to the Tuning Quadrupole MQT [8].

\section{Pre-Stress by Scissor-Laminations and Shrink-Fit}

Mounting consecutive shrinking rings around the yoke laminations generates the pre-stress in the coil. This assembly method is very fast and does not need an expensive press. To be able to transfer the force from the shrinking cylinders to the coil, the laminations have been made slightly eccentric ("Scissor laminations") and have been placed in different orientations each lamination transmitting force over its wide side only [7]. This system, where the shrinking rings are not mounted directly around the coils but further out around the yoke, has several advantages. 1) The thermal shrinking is much easier to achieve because it is easier to obtain the necessary assembly play at a larger radius. 2) The pre-stress at cold is enhanced thanks to the strong contraction of the large diameter shrink ring and the low contraction of the intermediate iron yoke. 3) The coil does not suffer from high shrinking temperatures being protected by the iron yoke.

\section{Keyed in Support Structure}

The magnet modules consisting of a coil assembly, yoke and shrinking cylinders are complete magnets. To obtain a double aperture magnet, the modules are mounted in pairs in support structures made of iron laminations and which serve for 1) alignment 2) magnetic shielding and 3) as filler for the helium vessel. This design allows to test at the manufacturers the room temperature field quality as well as the $4.2 \mathrm{~K}$ training on single mod- ules. The precise alignment in the support structure is obtained by means of keys.

\section{Cost}

Presently contracts have been placed for all the corrector magnets through tendering procedures all over Europe. A study, made to see if the cost could be related to any of the magnet parameters, showed that surprisingly the cost correlates best with the length of the magnets. Fig. 6 shows an area that covers the cost of the modules of the 8 magnet types that have been ordered in numbers greater than 100. The costs are ex-works and include materials, work, tooling, inspection, magnetic measurement at room temperature and a training test on each magnet module at $4.2 \mathrm{~K}$. It does not include the support structures. The cost appears to be relatively independent of the wire type, the thickness of the coils and the number of the coils per magnet, the latter being a function of the multipole type of magnet. The cost of the superconducting wire represents typically $10 \%$ of the total magnet cost.

\section{CONCLUSIONS}

The different techniques described in this paper have been successfully developed and are now applied on a large scale to the series production of corrector magnets. They result in slim and powerful magnets that are produced at a reasonable cost of about 10 kEuro per meter of magnet independent of the multipole type.

\section{ACKNOWLEDGMENT}

The authors acknowledge the encouragement of T. Taylor and N. Siegel; the design effort of G. Trinquart, G. Laurent, H. Meyer, H. Durand, G. Dogru, and T. Strauli of the EST division; and the test work of their colleagues L. Walckiers, R. Senis, Ch. Giloux, and P. Viret of the MTA group.

\section{REFERENCES}

[1] J. Salminen and A. Ijspeert, "Experience with different constructions of superconducting corrector magnets for LHC," in EPAC-98 Stockholm, Sweden, 1998.

[2] M. Allitt et al., "Further development of the sextupole and decapole spool corrector magnets for the LHC," in MT-16, 1999.

[3] J. Salminen, A. Ijspeert, and A. Puntambekar, "Superconducting sextupole corrector magnet for the LHC main dipoles," in EPAC-96 Sitges, Spain, June 1996.

[4] A. Ijspeert et al., "Construction and tests of a model of the LHC superconducting corrector magnet MDSBV," in MT-12 Leningrad, June 1991.

[5] M. Allitt, "Development of prototype superconducting tuning quadrupole magnets for LHC," in MT-17 Geneva, Switzerland.

[6] F. Toral et al., "Design and fabrication of a superconducting trim quadrupole for the LHC," in 4th Europ. Conf. Appl. Superconduct., ser. Inst. of Physics Conf. Series Sitges, 1999, vol. 1, pp. 1195-1198.

[7] A. Ijspeert and J. Salminen, "Superconducting coil compression by scissor laminations," in EPAC-96 Sitges, Spain, June 1996.

[8] K. Preuschoff, Diploma Thesis, Technische Universitaet Berlin, June 2000 